\title{
Herkunftseffekte und Gerechtigkeitserleben beim Übergang von der Primarschule in die Sekundarstufe I
}

\section{Caroline Biewer ${ }^{1}$, Christian Wandeler ${ }^{1}{ }^{2}$ und Franz Baeriswyl ${ }^{1}$}

Im deutschsprachigen Teil des Kantons Freiburg/CH kommt ein multikriteriales Übertrittsverfahren beim Übergang von der Primarschule in die Sekundarstufe I zum Einsatz. Nebst Schulnoten, den Einschätzungen der kognitiven Fähigkeiten durch die Lehrpersonen sowie der Zuweisungsempfehlung der Lehrpersonen und der Eltern wird zusätzlich eine Vergleichsprüfung in Deutsch und Mathematik durchgeführt. In zwei Vollerhebungen wurden die Lernenden, deren Eltern sowie die Lehrpersonen der Übertrittsjahrgänge 2009 und 2010 ausführlich befragt. Die Daten erlaubten eine empirische Trennung der primären von sekundären Herkunftseffekten und charakterisieren somit den übertrittsrelevanten Einfluss sozialer Disparitäten. Auch unter Kontrolle der Leistung, der Noten und der Zuweisungsempfehlungen der Eltern und der Lehrpersonen blieben die Einflüsse der Herkunft bestehen. Die sekundären Herkunftseffekte spiegelten sich nicht in der Gerechtigkeitswahrnehmung der Eltern mit tieferem sozioökonomischem Hintergrund wider. Es handelte sich tendenziell um Eltern aus höheren Sozialschichten, die Ungerechtigkeiten beim Übergang wahrnahmen. Generell kann die von den Eltern wahrgenommene Gerechtigkeit des Deutschfreiburger Übertrittsverfahrens vor allem durch lehrpersonbezogene Variablen erklärt werden.

\section{Einleitung}

In den vergangenen Jahren hat sich im deutschsprachigen Raum ein Wort durchgesetzt, welches den Übergang von der Primarschule in die Sekundarstufe I auf besondere Art und Weise charakterisiert - das Grundschulabitur. Gemeint sind hiermit die hohen Anforderungen seitens der Eltern und Lehrpersonen, die an die jungen Schülerinnen und Schüler $(\mathrm{SuS})$ im letzten Primarschuljahr gestellt werden, um einen bestmöglichen Übertritt in die höchstmögliche Anschlusslösung - nämlich in das Gymnasium - zu erhalten. Die wissenschaftliche Forschung beschäftigt sich bereits seit geraumer Zeit mit Bildungsverläufen, insbesondere mit dem Übergang von der Primarschule in die Sekundarstufe I. 
Hier zeigt sich allerdings, dass neben den Leistungen der Kinder Merkmale der sozialen Herkunft bedeutsame Einflussgrössen für die individuellen Bildungsverläufe darstellen (u. a. Baumert \& Schümer, 2001; Bos et al., 2003; Baeriswyl et al., 2006; Trautwein et al., 2008; Jonkman et al., 2010).

Inwiefern soziale Ungleichheiten von den am Übergang beteiligten Kindern, deren Eltern und Lehrkräften bewusst wahrgenommen und als Ungerechtigkeit betrachtet werden, ist bisher nur wenig erforscht. Es ist bekannt, dass Eltern den Einfluss des sozioökonomischen Status wahrnehmen und dass dieses Wissen, auf das generelle Gerechtigkeitserleben bezogen, auf Übergangssituationen einwirkt (Baeriswyl et al., 2013). Allerdings bleibt offen, ob sich das Gerechtigkeitserleben je nach Schichtzugehörigkeit unterscheidet.

Die wahrgenommene Gerechtigkeit ist aus verschiedenen Gründen relevant. Erstens sind übertrittsbezogene Entscheidungsprozesse komplex und beeinflusst durch den sozialen Hintergrund. Aggregiert betrachtete Entscheidungsmuster können von den Betroffenen im Einzelfall durchaus als freie und sozial gerechte Entscheidung erlebt werden. Zweitens ist die Bildungsforschung geprägt von den (schul)bildungsnahen Werten von Bildungsforschern. Schulische und universitäre Bildung wird implizit und teils explizit der beruflichen Bildung vorgezogen. Daher werden Zuweisungsentscheidungen oder Empfehlungen, welche in Richtung des beruflichen Bildungswegs weisen, zum Teil als sozial ungerecht interpretiert. Gerade in der Schweiz ist der berufliche Bildungsweg jedoch für Individuen und Gesellschaft ein Erfolgsmodell und hat seit der Öffnung des Bildungssystems, beispielsweise durch die Einführung der Berufsmatura, auch die nötige Flexibilität für spätere Umorientierungen. Dass nicht nur gymnasiale Bildungswege erfolgreiche Karrierechancen bieten, kann einen Einfluss auf die Wahrnehmung der Gerechtigkeit des Übertrittsverfahrens an sich und dessen Konsequenzen haben. Drittens sind das Gerechtigkeitserleben der Betroffenen und die Wahrnehmung in der Bevölkerung interessant für die Bildungspolitik in einer Demokratie, weil die Wahrnehmung der Realität entscheidend für zukünftige wegweisende bildungspolitische Entscheidungen ist. Zum Beispiel kann ein Bildungssystem, welches objektiv fördernd für die Chancengleichheit ist, von darin agierenden Personen als fördernd, indifferent oder gar der Chancengleichheit abträglich wahrgenommen werden. Entsprechend kann auch die Wahrnehmung eines objektiv sozial ungerechten Bildungssystems erwartungswidrig ausfallen. Es ist deshalb entscheidend, dass die Bildungsforschung nicht nur aus wissenschaftlicher Sicht objektive, sondern auch subjektive Wahrnehmungen der Realität erforscht. Der Öffentlichkeit sollte die Datengrundlage geliefert werden, um eine differenziertere Meinungsbildung zu ermöglichen. So können chancengleichheitsfördernde Prozesse bewahrt oder eingeführt werden und Aspekte des Bildungssystems, die nicht förderlich sind, jedoch eventuell sogar als förderlich wahrgenommen werden, sachlich diskutiert und reformiert werden.

Der vorliegende Beitrag untersucht anhand einer Gesamterhebung der Schülerjahrgänge von 2009 und 2010 des deutschsprachigen Teils des Kantons 
Freiburg die Herkunftseffekte und die von den Eltern wahrgenommene Gerechtigkeit des Deutschfreiburger Modells zum Übergang von der Grundschule in die Sekundarstufe I. Im nächsten Abschnitt wird der theoretische Hintergrund dargestellt und im dritten Abschnitt die Fragestellung im Detail erläutert. Im darauf folgenden vierten Punkt werden die institutionellen Rahmenbedingungen für den Bildungsübergang erklärt. Die Darstellung über Daten, Verfahren und Variablen erfolgt im fünften Abschnitt, während unter Punkt sechs die Resultate präsentiert werden. Im siebten Abschnitt werden abschliessend die Befunde diskutiert.

\section{Theorie}

Es besteht ein starkes öffentliches Interesse an herkunftsbezogenen Unterschieden in Schullaufbahnen. Im Sinne der Chancengleichheit zitieren die Medien die Ergebnisse jener Studien, die belegen, dass die soziale Herkunft für den Schulerfolg mitentscheidend ist. Wie zum Beispiel, dass Kinder aus bildungsfernen Elternhäusern in Schulen ungerecht behandelt werden oder, dass bei gleicher Leistung Schüler, in sozial schwierigen Verhältnissen aufwachsend, schlechtere Noten erhalten als ihre Schulkameraden aus den bildungsnäheren Schichten. Nur selten wird bei der Darstellung dieser Herkunftseffekte in den öffentlichen Medien verdeutlicht, dass in der wissenschaftlichen Literatur derzeit zwischen primären und sekundären Disparitäten, im Sinne des Modells von Boudon (1974), unterschieden wird (u. a. Becker \& Schubert, 2011; Becker, 2011).

Primäre Herkunftseffekte bezeichnen tatsächliche, durch die soziale Herkunft bedingte Leistungsunterschiede. Sie können auf Herkunftsmerkmale wie den sozioökonomischen Status, die Bildungsnähe der Erziehungsverantwortlichen oder auf ihre kulturellen Betätigungen zurückgeführt werden. Boudon (1974) selbst geht von einer Interaktion von Anlage und sozialer/materieller Umwelt aus, so dass den Kindern aus bildungsfernen und sozial weniger begünstigten Familien weniger Ressourcen zur Förderung und Entwicklung zur Verfügung stehen. Die Sozialisationsbedingungen können auch zu einer nicht optimalen Nutzung der familiären Lernumgebung führen. Beispielhaft seien hier die von Becker (2004) identifizierten, auf die Sozialschicht bezogenen, habitualisierten Lerngewohnheiten genannt. Dieser herkunftsbezogene Sozialisationseffekt erklärt Unterschiede zwischen den Sozialschichten, die sich in der Vermittlung von Sprachkultur, in der Lern- und Bildungsmotivation sowie dem selbstregulierten Handeln und Lernen widerspiegeln. Sowohl bei der individuellen Leistung als auch bei der Ressourcenausstattung führen primäre Herkunftseffekte zu einer ungleichen Ausgangsverteilung bei den Kindern (Maaz et al., 2006), so dass Kinder aus bildungsfernen Schichten bereits mit einem geringeren Vorwissen in die Schulkarriere starten.

Unter die sekundären Herkunftseffekte werden jene soziale Disparitäten gefasst, die ausgehend von schichtspezifischen Bildungsaspirationen und 
Entscheidungsverhalten, unabhängig vom Kompetenzniveau der Kinder, auftreten (Maaz et al., 2006; Maaz et al., 2009; Maaz \& Nagy, 2009). Maaz et al. (2006, S. 303) bezeichneten den sekundären Herkunftseffekt als «kumulativen Effekt der verinnerlichten Sozialschichtzugehörigkeit» und verwiesen darauf, dass dieser sich auf die «Social Position Theory» nach Keller und Zavallione (1964, zitiert nach Maaz et al. 2006) zurückführen lässt. Neben den, auf differenzielles Entscheidungsverhalten von Eltern und Kindern rückführbaren, Unterschieden in den Bildungsverläufen werden zunehmend auch schichtspezifische Zuschreibungen bei den Lehrpersonen betrachtet. Diese spiegeln sich in sozialstatusabhängigen Beurteilungen oder Zuweisungsempfehlungen wider (Maaz \& Nagy, 2009, Baeriswyl et al., 2006; Ditton, 2008). Baumert et al. (2003) erklärten diesen Effekt als eine von der Lehrperson antizipierte zukünftige Unterstützungsleistung der Eltern. Kinder aus bildungsfernen Milieus würden demnach für höhere Schulformen nicht ausreichende Unterstützung von zu Hause erhalten, um diese mit Erfolg zu durchlaufen.

Insbesondere für den Übergang von der Primarschule in die Sekundarstufe I konnten die Effekte der sozialen Herkunft bisher mehrfach gezeigt werden (u. a. Baumert \& Schümer, 2001; Becker, 2000, 2003; Ditton, 2007; Ditton \& Krüsken, 2006; Ditton et al., 2005; Maaz \& Nagy, 2009; Baumert et al. 2010; Baeriswyl et al. 2006; Neuenschwander \& Grunder, 2010; Baeriswyl et al., 2011). Zusammenfassend bedeuten die Ergebnisse, dass Kinder aus bildungsfernen Familien, verglichen mit ihren Schulkameraden aus bildungsnäheren Familien, (1) niedrigere schulische Leistungen und Noten erlangen, (2) bei vergleichbaren Leistungen von Lehrpersonen schlechter beurteilt werden, (3) auch unter Kontrolle der schulischen Leistungen und der Noten geringere Chancen haben, eine Gymnasialempfehlung zu erhalten, und (4) unter Kontrolle der Leistung, die Eltern ihre Kinder seltener auf ein Gymnasium schicken (Maaz et al., 2011).

Die durch die soziale Herkunft bedingten Disparitäten zwischen Kindern aus bildungsfernen und bildungsnahen Familien waren der Ausgangspunkt für eine ausgeprägte und bis heute andauernde Diskussion um Bildungsgerechtigkeit und Chancengleichheit. Als Folge der PISA-Ergebnisse wurde besonders der sekundäre Herkunftseffekt als unvereinbar mit den Prinzipien der leistungsbezogenen Verteilungsgerechtigkeit dargestellt (Baumert et al., 2010). Bereits in den 70er Jahren bestand die Sollensforderung, dass Bildungschancen einzig aufgrund der Leistungsfähigkeiten des Kindes vergeben werden sollten (u. a. Fend, 1974). Allerdings ist dies eine Gerechtigkeitsforderung, die unabhängig vom Gerechtigkeitserleben der beteiligten Akteure bei Übergangsentscheidungen gestellt wird.

\section{Gerechtigkeitserleben im Schulkontext}

Gerechtigkeit wird als soziales Konstrukt aufgefasst (Maier et al., 2007), als eine nicht materielle latente Grösse, die in menschlichen Interaktionen enthalten ist (Peter et al., 2013). Während sich Disziplinen wie Philosophie, Theologie und Rechtswissenschaften damit auseinandersetzen, allgemeingültige Massstäbe 
zur Lösung gerechtigkeitsbezogener Probleme aufzustellen und ethisch zu begründen, liegt der Fokus der Psychologie auf dem „naiven Rechts- und Gerechtigkeitsempfinden der betroffenen Menschen" (Schmitt, 1993, S. 1). Die Bewertung von gerecht und ungerecht findet durch subjektive Informationsverarbeitungsprozesse im sozialen Kontext statt. Daher sind Gerechtigkeitskognitionen und Gerechtigkeitsurteile von interindividuellen Unterschieden abhängig, z. B. von der Persönlichkeit, von Erfahrungen oder von Bevorzugung (Mikula, 1980; Peter et al., 2013).

Neben Wissenschaftlern, die sich intensiv mit der Gerechtigkeit von Bildungssystemen auseinandersetzen, tun dies auch die im Schulumfeld agierenden Personen. Schülerinnen und Schüler haben den Anspruch, von ihren Lehrpersonen gerecht beurteilt zu werden (Tyler, 1962; Fischer, 2007) und auch den Lehrpersonen ist es wichtig, bei Bewertungen oder Sanktionen gerecht vorzugehen (Kanders, 2000). Erlebte Ungerechtigkeit führt bekanntermassen zu einem Gefühl des Unbehagens. Die besondere Bedeutung der Lehrpersongerechtigkeit für die Schülerinnen und Schüler konnte bereits wiederholt herausgestellt werden (Dalbert \& Stoeber, 2006; Dalbert, 2013). Im Detail zeigte Dalbert (2011), dass das individuell und subjektiv erlebte gerechte Lehrerhandeln positive Einflüsse auf regelkonformes Verhalten der Schülerinnen und Schüler, das Unterrichtsklima, das Belastungserleben und das Exklusionsempfinden hat.

Die einflussreiche Rolle der Lehrpersonen für das Gerechtigkeitserleben der Eltern bei Übertritten konnten Baeriswyl et al. (2013) deutlich aufzeigen. Bezüglich der elternperzipierten Gerechtigkeit des Übertrittsverfahrens konnte gezeigt werden, dass die globale Übertrittsgerechtigkeit stark von lehrpersonbezogenen Merkmalen abhängig ist. Je höher Eltern die Vorbereitungsgüte durch die Lehrperson auf eine versetzungsrelevante Prüfung einschätzten, desto höher wurde auch die Gerechtigkeit des gesamten Übertrittsverfahrens eingestuft. Die gleiche Auswirkung zeigte die elternperzipierte Beurteilungsgerechtigkeit durch die Lehrperson. Hatten Eltern den Eindruck, dass die Klassenlehrperson ihr eigenes Kind sowie die anderen Kinder in der Klasse gerecht bewertete, so fiel auch die Beurteilung der Gerechtigkeit des Übertrittsverfahrens höher aus. Das Gerechtigkeitserleben wird aber auch von herkunftsspezifischen Variablen beeinflusst. Der von den Eltern wahrgenommene Einfluss des sozioökonomischen Status ging mit einer niedrigeren Beurteilung der Gerechtigkeit des Übertrittsverfahrens einher. Weiter berichtete Baeriswyl (2013), dass sich die Eltern über die Existenz sozialer Herkunftseffekte bewusst sind, aber trotzdem grossmehrheitlich das Übertrittsverfahren als gerecht beurteilen. Hinsichtlich des Bewusstseins dafür, dass soziale Disparitäten durch Schule und Familie reproduziert werden (u. a. Ditton et al., 2005), sollte das Erleben und Beurteilen der Eltern detaillierter betrachtet werden. 


\section{Fragestellung}

Anlehnend an die bisherige Forschung zu sozialen Disparitäten am Übergang von der Primarschule in die Sekundarstufe I, sollen in vorliegender Arbeit die primären und sekundären Herkunftseffekte auf den definitiven Zuweisungsentscheid dargestellt werden. Als Leistungsmass dient die obligatorische Vergleichsprüfung. Die Elternempfehlung, die Lehrpersonempfehlung, und die Noten fungieren ebenfalls als Prädiktoren der definitiven Zuweisung. Es ist davon auszugehen, dass Schülerinnen und Schüler aus bildungsfernen Familien bei gleicher Leistung in der Vergleichsprüfung tiefere Zuweisungen erhalten als Kinder aus bildungsnahen Familien. Die Analysen sollen auch getrennt für die Lehrpersonempfehlung und die Elternempfehlung berechnet werden, um Hinweise auf unterschiedliche Vermittlung der Herkunftseffekte zu erlangen.

Anknüpfend an die Forschungsarbeit zum elterlichen Gerechtigkeitserleben des Übertrittssystems in Freiburg $(\mathrm{CH})$ von Baeriswyl et al. (2013), ist eine weitere Prüfung des Modells vorgesehen. Ziel hierbei ist es, sowohl die globale Übertrittsgerechtigkeit als auch die lehrpersonbezogenen Merkmale aufzuklären.

Wie bereits beschrieben, hängen Gerechtigkeitserleben und -beurteilung von verschiedenen personspezifischen und situationsspezifischen Faktoren ab. Um ein umfassendes Modell des Übertrittssystems darzustellen, werden die zuweisungsrelevanten Masse (Noten, Prüfung und Zuweisungsempfehlung) in ein Strukturgleichungsmodell aufgenommen. Die elterliche Zufriedenheit mit der Zuweisung und die elternperzipierte Beurteilungsgerechtigkeit der Lehrperson haben sich als wichtige Einflussfaktoren für eine globale Beurteilung der Gerechtigkeit des Übertrittsverfahrens herausgestellt (Baeriswyl et al., 2013). Es wird nun vermutet, dass Noten, Prüfungsergebnisse und Übergangsempfehlungen ebendiese Zufriedenheits- und Gerechtigkeitsmasse beeinflussen. Dem Grad an Übereinstimmung zwischen der Elternempfehlung und der Lehrpersonempfehlung wird ein hoher gerechtigkeitsrelevanter Stellenwert beigemessen. Wenn Eltern eine höhere Empfehlung für den Übergang in die Sekundarstufe I abgeben als die Lehrpersonen, sollten Eltern die Beurteilungsgerechtigkeit der Lehrperson niedriger einstufen und auch weniger zufrieden mit dem definitiven Zuweisungsentscheid sein. Es ist nunmehr bekannt, dass Noten und Zuweisungsempfehlungen durch den sozioökonomischen Status der Eltern beeinflusst werden (Baeriswyl et al., 2006) und Eltern diese sozialen Disparitäten wahrnehmen. Demensprechend soll detailliert aufgeschlüsselt werden, wie der sozioökonomische Status auf die Gerechtigkeitswahrnehmung des Übertrittsverfahrens wirkt. 


\section{Das übertrittsverfahren im deutschsprachigen Teil des Kantons Freiburg}

Im deutschsprachigen Teil des schweizerischen Kantons Freiburg wird für den Übergangsentscheid von der Primarschule in die Sekundarstufe I ein mehrkriteriales Verfahren eingesetzt. Es umfasst eine standardisierte, auf einem Leistungstest basierende Leistungsstandbeurteilung, eine Berücksichtigung der Motivation und des Arbeitsverhaltens der Schülerin und des Schülers sowie obligatorische, den Zuweisungsentscheid betreffende Beratungsgespräche mit den Eltern.

Zu Beginn des zweiten Semesters des 6. Schuljahres gaben die Lehrpersonen globale Zuweisungsempfehlungen für die Schülerinnen und Schüler für eine der Abteilungen der Orientierungsschule ab: der Progymnasialabteilung mit erweiterten Leistungsansprüchen, der Allgemeinen Sekundarabteilung mit regulären Ansprüchen und der Realabteilung mit Grundansprüchen. Eine weitere Option stellte die individuelle Abklärung des Besuchs der Werkklasse dar. Zusätzlich machten die Lehrpersonen Angaben zu kognitiven Fähigkeiten, zur Motivation und zum Lern- und Arbeitsverhalten der jeweiligen Schülerinnen und Schüler. Die Empfehlung der Lehrperson wurde den Eltern in einem persönlichen Beratungsgespräch mitgeteilt und deren Zustandekommen erläutert. Im Anschluss waren die Eltern ebenfalls dazu aufgefordert, eine Zuweisungsempfehlung für ihr Kind in schriftlicher Form abzugeben. Anfang März wurde eine standardisierte Vergleichsprüfung in den Fächern Mathematik und Deutsch durchgeführt, an der alle Schülerinnen und Schüler des 6. Schuljahrs teilzunehmen hatten. Die Aufgabeninhalte beziehen sich auf den Lehrplan der fünften und einen Teil der sechsten Klasse, werden jährlich von unbeteiligten Primarund Sekundarlehrpersonen neu entwickelt und in einem anderen Kanton mit gleichem Lehrplan vorgetestet. Die Übertrittsprüfung wird im Primarklassenzimmer unter Aufsicht einer Sekundarlehrperson am kantonalen Testtag durchgeführt, an der abnehmenden Orientierungsschule von Fachlehrpersonen korrigiert und für ganz Deutschfreiburg zentral ausgewertet.

Um einen definitiven Zuweisungsentscheid zu treffen, wurde nun zuerst die Lehrpersonempfehlung mit dem Resultat der Vergleichsprüfung verglichen. Stimmten beide Elemente überein, d. h., legten sie eine Zuweisung in die gleiche Abteilung nahe, wurde der Entscheid direkt gefällt und schriftlich an die Eltern zugestellt. Im Fall der Nicht-Übereinstimmung wurde der Entscheid vom Schuldirektor getroffen. Hierfür führte dieser eine detaillierte Analyse der Daten durch und führte mit der Lehrperson und den Eltern ein lösungsorientiertes Gespräch. Gegen den definitiven Zuweisungsentscheid des Schuldirektors konnten Eltern einen schriftlichen Rekurs bei der Direktion für Erziehung einlegen. 


\section{Methode}

\section{Befragung}

Zusätzlich zu den regulären Daten des Übertrittsverfahrens wurden die Jahrgänge 2009 und 2010 umfassend zum Übergang schriftlich befragt. Sowohl die Schülerinnen und Schüler als auch deren Eltern und Lehrpersonen nahmen an der Erhebung teil. Die zentralen Elemente der Befragung waren: Migrationshintergrund der Schülerinnen und Schüler, Familiensprache, kulturelle und soziökonomische Hintergrundvariablen, Hausaufgabenverhalten, die wahrgenommene Gerechtigkeit des Übertrittsverfahrens sowie die fremdperzipierte Beurteilungsgerechtigkeit und Vorbereitungskompetenz der Lehrpersonen. Die Datenerhebung fand nach der Bekanntgabe der definitiven Zuweisungsentscheide und nach Ablauf der Rekursfrist statt.

\section{Stichprobe}

Aus der Vollerhebung der Kohorten der Jahre 2009 und 2010 ergab sich eine Stichprobe, bestehend aus 1685 Schülerinnen und Schülern. Diese sind 94 Klassen zuzuordnen. Insgesamt gab es 83 Klassenlehrpersonen, da 11 von ihnen in beiden Jahrgängen mit einer Klasse einhergingen. Die Teilnehmerzahl der Eltern beträgt 1511 Personen. Dies bedeutet eine Rücklaufquote von 90 Prozent. Kinder mit einer Zuweisung in die Werkklasse wurden in die Analysen nicht einbezogen, da sie nicht an der Vergleichsprüfung teilnahmen.

\section{Variablen und Instrumente}

In der vorliegenden Studie wurde der sozioökonomische Status durch den internationalen sozioökonomischen Index - ISEI (Ganzeboom \& Treiman, 1996) - abgebildet. Die Angaben bezüglich des Berufes des Vaters und der Mutter wurden hierbei in das vorgegebene Schema übertragen und der höhere Wert als Indikator für den sozioökonomischen Status des Kindes herangezogen.

Die Zuweisungsempfehlungen der Eltern und der Lehrpersonen besitzen die gleichen Abstufungen. Die Empfehlungen in die Progymnasialabteilung (A), in die allgemeine Sekundarabteilung (B) und die Realabteilung (C) konnten auch als bedingte Empfehlung abgegeben werden (A bedingt \& B bedingt), daher handelt es sich um eine fünfstufige Variable.

Aus der Differenz der Lehrpersonempfehlung und der Elternempfehlung wurde das Übereinstimmungsmass der Empfehlung gebildet $(N=1616$; Eltern > Lehrperson: $n=286$; Eltern $=$ Lehrperson: $n=1306$; Eltern $<$ Lehrperson: $n=24)$.

Die definitive Zuweisung ist dreistufig; 1 Realabteilung (C), 2 allgemeine Sekundarabteilung (B) und 3 Progymnasialabteilung (A).

Der in dieser Untersuchung verwendete Notendurchschnitt setzte sich aus den Halbjahreszeugnisnoten der Fächer Mathematik und Deutsch zusammen (6 = sehr gut, 5 = gut, 4 = genügend und 3,2,1 = ungenügend). 
Im Rahmen des Übertrittsverfahrens wurde ein standardisierter Mathematikund Deutschtest (Übertritts- bzw. Vergleichsprüfung) mit jeweils 50 Punkten administriert, so dass insgesamt maximal 100 Punkte erreicht werden konnten.

Folgende elternperzipierte Skalen wurden eingesetzt: Die Skala elternperzipierte Übertrittsgerechtigkeit bestand aus 4 vierstufigen Items («stimmt gar nicht» bis «stimmt genau», z. B.: «Das Übertrittsverfahren ist gerecht», «Die Zuweisung ist für alle SuS gerecht») mit einem Cronbach's Alpha $\alpha=0,88$. Elternperzipierte Beurteilungsgerechtigkeit der Lehrperson bestand aus 2 vierstufigen Items («Die Lehrperson gibt gerechte Übetrittsempfehlungen», «Die Lehrperson gibt gerechte Noten») mit $\alpha=0,80$. Elternperzipierte Vorbereitungskompetenz der Lehrperson umfasste 2 fünfstufige Items («Die Lehrperson bereitete mein Kind sehr gut auf die Vergleichsprüfung vor» «Die Lehrperson bereitete die Klasse sehr gut auf die Vergleichsprüfung vor») und erreichte eine Reliabilität von $\alpha=0,92$. Die Skala elternberichtete Zufriedenheit mit Zuweisung setzte sich aus 5 fünfstufigen Items zusammen («Ich trage den Zuweisungsentscheid voll mit», "Ich bin mit der Zuweisung zufrieden», "Ich hätte mir eine andere Zuweisung erhofft») mit $\alpha=0,86$.

\section{Statistisches Vorgehen}

Zur Überprüfung der Modelle wurde die Software Mplus 6.0 (Muthén \& Muthén, 2011) verwendet. Um die definitive Zuweisung vorherzusagen, wurden ordinale logistische Regressionsanalysen durchgeführt. Wie in der empirischen Schulforschung üblich, weisen die vorliegenden Daten eine hierarchische Struktur auf, die in der Datenanalyse nach Möglichkeit berücksichtigt werden sollte. Im vorliegenden Fall sind Schülerinnen und Schüler in den Grundschulklassen geschachtelt. Wird die Schachtelung der Daten nicht berücksichtigt, kann es zu einer zu liberalen Signifikanztestung kommen. Derzeit sind die gängigen Statistikpakete noch nicht in der Lage, die hier vorliegende komplexe Schachtelung der Daten in Verbindung mit einer ordinalen logistischen Regressionsanalyse adäquat zu modellieren. Deswegen wird hier pragmatisch vorgegangen, indem auf eine Berücksichtigung der Schachtelung verzichtet und stattdessen das Signifikanzniveau auf $p<.025$ festgelegt wird. Für die Vorhersage des Gerechtigkeitserlebens wurde ein Pfadmodell spezifiziert, bei welchem die Schachtelung der Daten durch eine Korrektur der Standardfehler mittels der Funktion «type=complex" berücksichtigt wurde. Ebenfalls wurden Variablen, sofern möglich, latent modelliert. Die Anpassungsgüte des Strukturgleichungsmodells erwies sich als adäquat (siehe Abb. 1). Für den Umgang mit fehlenden Daten wurde die in Mplus implementierte "Full Information Maximum Likelihood (FIML)»-Methode verwendet (Enders \& Bandalos, 2001; Raykov, 2005). Die deskriptiven Analysen wurden mit dem Statistikpaket SPSS 21 gerechnet. 


\section{Ergebnisse}

\section{Gerechtigkeit des Zuweisungsentscheids}

Zur Aufklärung des definitiven Zuweisungsentscheids wurden fünf Modelle spezifiziert, die schrittweise folgende Variablen in die ordinalen Regressionsanalysen aufnahmen: Geschlecht, Sprache, ISEI, Notendurchschnitt, Vergleichsprüfung, Lehrperson- und Elternempfehlung (siehe Tab. 1).

In Modell 1.1 wurden die Variablen Geschlecht, Sprache, sozioökonomischer Status und Notendurchschnitt als Prädiktoren aufgenommen. Hierbei zeigten die Odds ratios (OR), dass die Chance, in die Progymnasialabteilung zugewiesen zu werden, 85-mal höher war, wenn der Notendurchschnitt um eine Standardabweichung zunahm. Mit einem um eine Standardabweichung höheren ISEI der Eltern war die Chance, in die höchste Abteilung zu kommen, rund 1,9-mal höher.

In Modell 1.2 wurde zusätzlich die Übertrittsprüfung einbezogen. Geschlecht, Sprache, Notenschnitt und Übertrittsprüfung waren signifikant. Auch bei gleichem ISEI, Notendurchschnitt und gleicher Übertrittsprüfung hatten Jungen gegenüber Mädchen eine nur 0,6-fach so grosse Chance, in die Progymnasialabteilung zu kommen. Der Notendurchschnitt und die Übertrittsprüfung zeigten sich ähnelnde Effekte. Jeweils um das 32-Fache stieg die Chance der Schülerinnen und Schüler mit einer um eine Standardabweichung höherem Notenschnitt oder höheren Punktzahl in der Übertrittsprüfung. Auch hier zeigte sich trotz der Kontrolle der Leistung ein Einfluss des sozioökonomischen Status auf den Zuweisungsentscheid. Ein um eine Standardabweichung höherer ISEI steigerte die Chance, in die höchste Abteilung zugewiesen zu werden, um das 2-Fache.

In Modell 1.3 wurde die Lehrpersonempfehlung hinzugefügt. Diese erwies sich als stärkster Prädiktor für die definitive Zuweisung, mit einer OR von 1829,5. Bei einer um eine Standardabweichung höheren Übertrittsprüfung stieg die Chance, in die Progymnasialabteilung zu gehen, um das 158-Fache. Der Notendurchschnitt hingegen steigerte die Chance auf die höchste Abteilung nur um das 2,2-Fache. Wieder zeigte sich der ISEI als signifikanter Einflussfaktor. Kinder mit einem um eine Standardabweichung höheren ISEI der Eltern hatten eine rund 1,7-mal grössere Chance, in die höchste Abteilung zugewiesen zu werden.

In Modell 1.4 wurde statt der Lehrpersonempfehlung die Elternempfehlung aufgenommen. Bei diesen Analysen zeigte sich wiederum ein Geschlechtereffekt. Jungen hatten gerade einmal eine 0,6-mal so grosse Chance, in die Progymnasialabteilung zu kommen, als Mädchen. Die Elternempfehlung hatte den höchsten Einfluss mit einer OR von fast 64. Bei einer hohen Punktzahl in der Übertrittsprüfung hatten Kinder eine 36-mal höhere Chance, in die Progymnasialabteilung zugewiesen zu werden, gegenüber Kindern, die um eine Standardabweichung schlechter abschnitten. Der Notendurchschnitt führt zu einer rund 


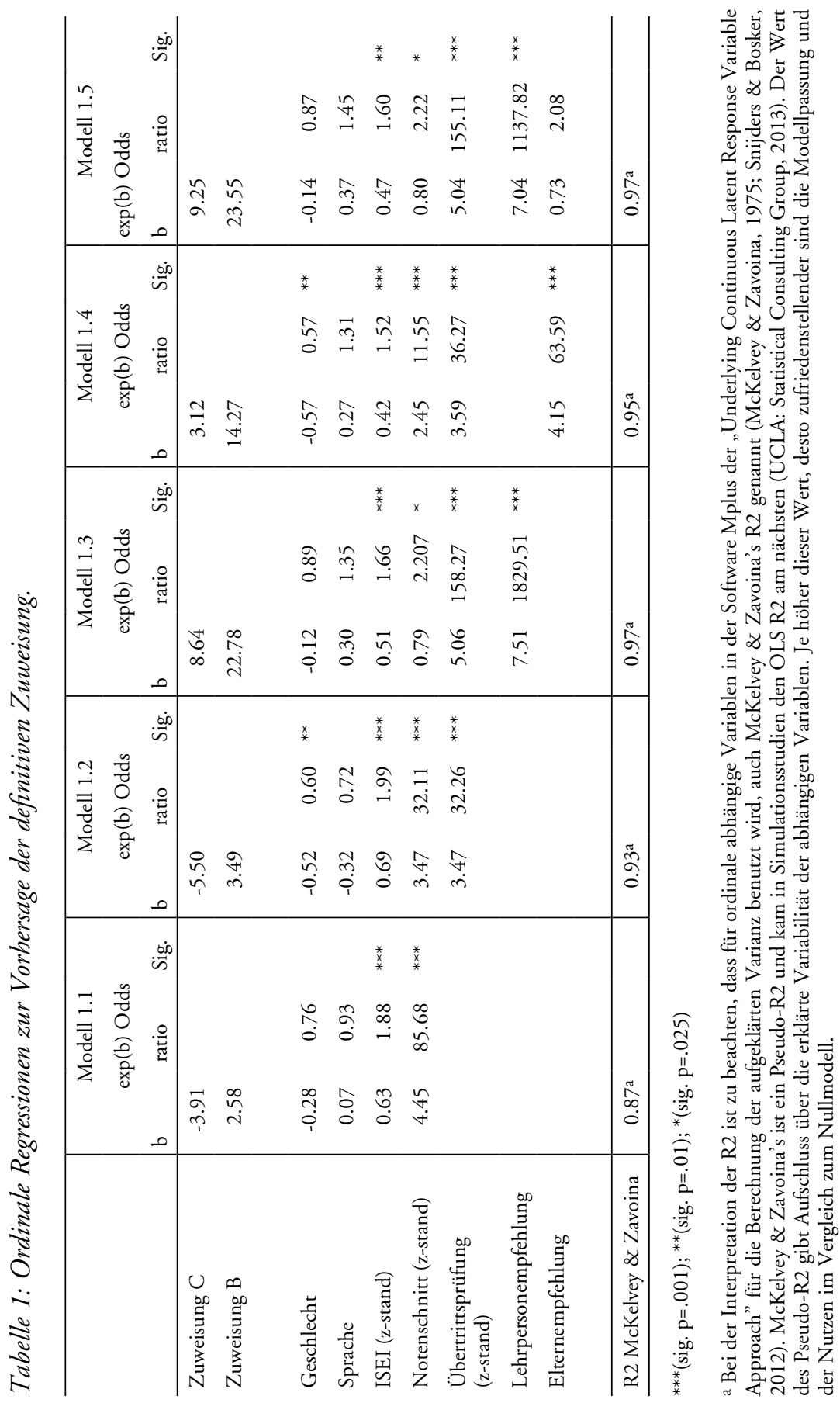


12-mal höheren Chance. Auch in diesem Modell zeigte sich der ISEI als signifikanter Prädiktor in gleicher Richtung wie in den vorher berichteten Modellen mit einer OR von 1,5 .

Das letzte Modell (1.5) beinhaltete alle Prädiktoren. Signifikante Effekte erzielten hierbei der ISEI, der Notendurchschnitt, die Übertrittsprüfung und die Lehrpersonempfehlung. Die höchste OR zeigte sich bei der Lehrpersonempfehlung mit 1138. Eine um eine Standardabweichung bessere Übertrittsprüfung führte zu einer 155-mal höheren Chance, in die Progymnasialabteilung zugewiesen zu werden. Ein um eine Standardabweichung höherer Notenschnitt verdoppelte die Chance eines Kindes, in die höchste Abteilung zu gelangen. Die Erhöhung des ISEI um eine Standardabweichung führte zu einer 1,6-fach grösseren Chance, in die Progymnasialabteilung zugewiesen zu werden.

\section{Gerechtigkeitserleben}

Die elternperzipierte Übertrittsgerechtigkeit korrelierte am höchsten mit den Variablen Beurteilungsgerechtigkeit, Zuweisungszufriedenheit, Vorbereitungskompetenz der Lehrperson und der Differenz der Empfehlungen. Eine negative Korrelation zeigte sich mit dem ISEI. Weiter ergaben sich hohe korrelative Zusammenhänge zwischen den Variablen Beurteilungsgerechtigkeit, Zuweisungszufriedenheit und Vorbereitungskompetenz der Lehrperson. Die definitive Zuweisung korrelierte am höchsten mit dem Notendurchschnitt und der Vergleichsprüfung, aber auch mit dem ISEI.

Das Pfadmodell zur Vorhersage der elternperzipierten Übertrittsgerechtigkeit bestand aus den zuweisungsrelevanten manifesten Variablen - wie etwa Übereinstimmung der Empfehlungen, Notendurchschnitt, Vergleichsprüfung und definitive Zuweisung - sowie den perzipierten Variablen Beurteilungsgerechtigkeit, Zuweisungszufriedenheit und Vorbereitungskompetenz der Lehrperson, die latent modelliert wurden (siehe Abb. 1). Aufgrund bekannter Zusammenhänge zwischen den zuweisungsrelevanten Variablen Übereinstimmung, Notenschnitt und Vergleichsprüfung, sowie zwischen den Beurteilungsvariablen Beurteilungsgerechtigkeit, Zuweisungszufriedenheit und Vorbereitungskompetenz (siehe Tab. 2), wurden im Modell Korrelationen zwischen diesen Variablen zugelassen.

Die primären Herkunftseffekte widerspiegeln sich in den positiven Effekten des ISEI auf den Notendurchschnitt und die Vergleichsprüfung. Die Übereinstimmung zwischen Eltern- und Lehrpersonempfehlung hängt nicht mit dem ISEI zusammen. Anzeichen für sekundäre Herkunftseffekte findet man im signifikanten direkten positiven Zusammenhang des ISEI mit dem definitiven Zuweisungsentscheid, unter der Kontrolle von Übereinstimmung, Notendurchschnitt und Vergleichsprüfung. Der Zuweisungsentscheid wiederum hatte einen relativ starken Effekt auf die Zuweisungszufriedenheit und einen weniger 


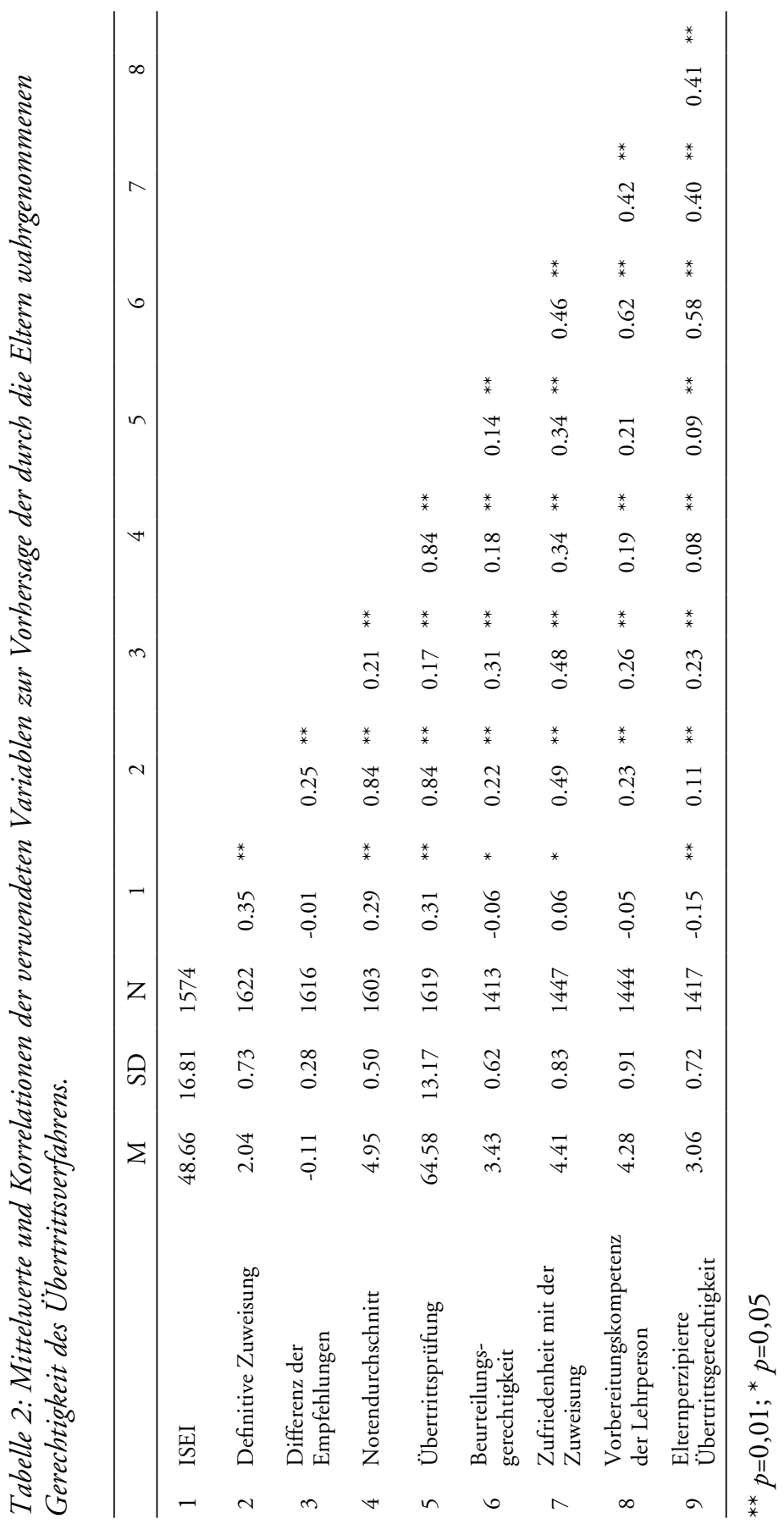


starken Effekt auf die Beurteilungsgerechtigkeit und die Vorbereitungskompetenz. Für alle Elternwahrnehmungen bestand ein schwacher, aber statistisch signifikant negativer Zusammenhang mit dem ISEI. Eine positive Ausprägung der Variable Übereinstimmung der Empfehlungen, wenn die Lehrperson die gleiche oder eine höhere Empfehlung ausgesprochen hatte als die Eltern, hatte positive Effekte auf die Beurteilungsgerechtigkeit, die Zuweisungszufriedenheit und die Vorbereitungskompetenz. Die Leistung an der Übertrittsprüfung hatte nur auf die Zuweisungszufriedenheit einen Einfluss, und dieser war negativ.

Die elternperzipierte Gerechtigkeit des Übertittsverfahrens hing am stärksten mit der wahrgenommenen Beurteilungsgerechtigkeit zusammen und stand in eher kleinem Zusammenhang mit der Zuweisungszufriedenheit. Die definitive Zuweisung und der ISEI hatten einen schwachen negativen Effekt auf die Übertrittsgerechtigkeit. Insgesamt kann das Modell 51 Prozent der elternperzipierten Gerechtigkeit des Übertrittsverfahrens aufklären.

\section{Diskussion}

Die Analysen erbrachten folgende wesentliche Befunde: Erstens treten sekundäre Herkunftseffekte des sozioökonomischem Status auf den definitiven Zuweisungsentscheid auf. Zweitens spielt für alle elternperzipierten Variablen die soziale Herkunft eine Rolle, und Eltern aus höheren Schichten schätzen Aspekte des Schulsystems wie Gerechtigkeit des Übertrittsverfahrens, Beurteilungsgerechtigkeit der Lehrperson und Vorbereitungskompetenz der Lehrperson tendenziell kritischer ein. Dies spiegelt sich auch in einer tendenziell tieferen Zuweisungszufriedenheit wider. Drittens kann die elternperzipierte Gerechtigkeitswahrnehmung des Übertrittsverfahrens vor allem durch die elternperzipierte Beurteilungsgerechtigkeit der Lehrperson vorhergesagt werden.

Diese Ergebnisse ergänzen vorliegende Befunde zur Wirkungsweise von Herkunftseffekten beim Übertritt (u. a. Baumert \& Schümer, 2001; Becker, 2000, 2003; Ditton, 2007; Ditton \& Krüsken, 2006; Ditton et al., 2005; Maaz \& Nagy, 2009; Baumert et al. 2010; Baeriswyl et al. 2006; Neuenschwander \& Grunder, 2010). Der Einfluss des sozioökonomischen Status der Eltern bei dem multikriterialen Übertrittsverfahren im Kanton Freiburg $(\mathrm{CH})$ bleibt sowohl unter Kontrolle der Lehrpersonempfehlung als auch der Elternempfehlung bei der Aufklärung des definitiven Zuweisungsentscheids bestehen. Auf Seiten der Lehrpersonen wie auch auf Seiten der Eltern gibt es sozialstatusabhängige Zuweisungsempfehlungen, die im Verständnis des sekundären Herkunftseffekts zu betrachten sind.

Neben der Tatsache, dass Kinder aus bildungsnäheren Familien eine grössere Chance haben, in die höchste Abteilung der Sekundarstufe I zugewiesen zu werden, zeigte sich auch, dass bei gleicher Elternempfehlung, gleichem Notendurchschnitt, gleicher Leistung in der Übertrittsprüfung und bei gleichem 


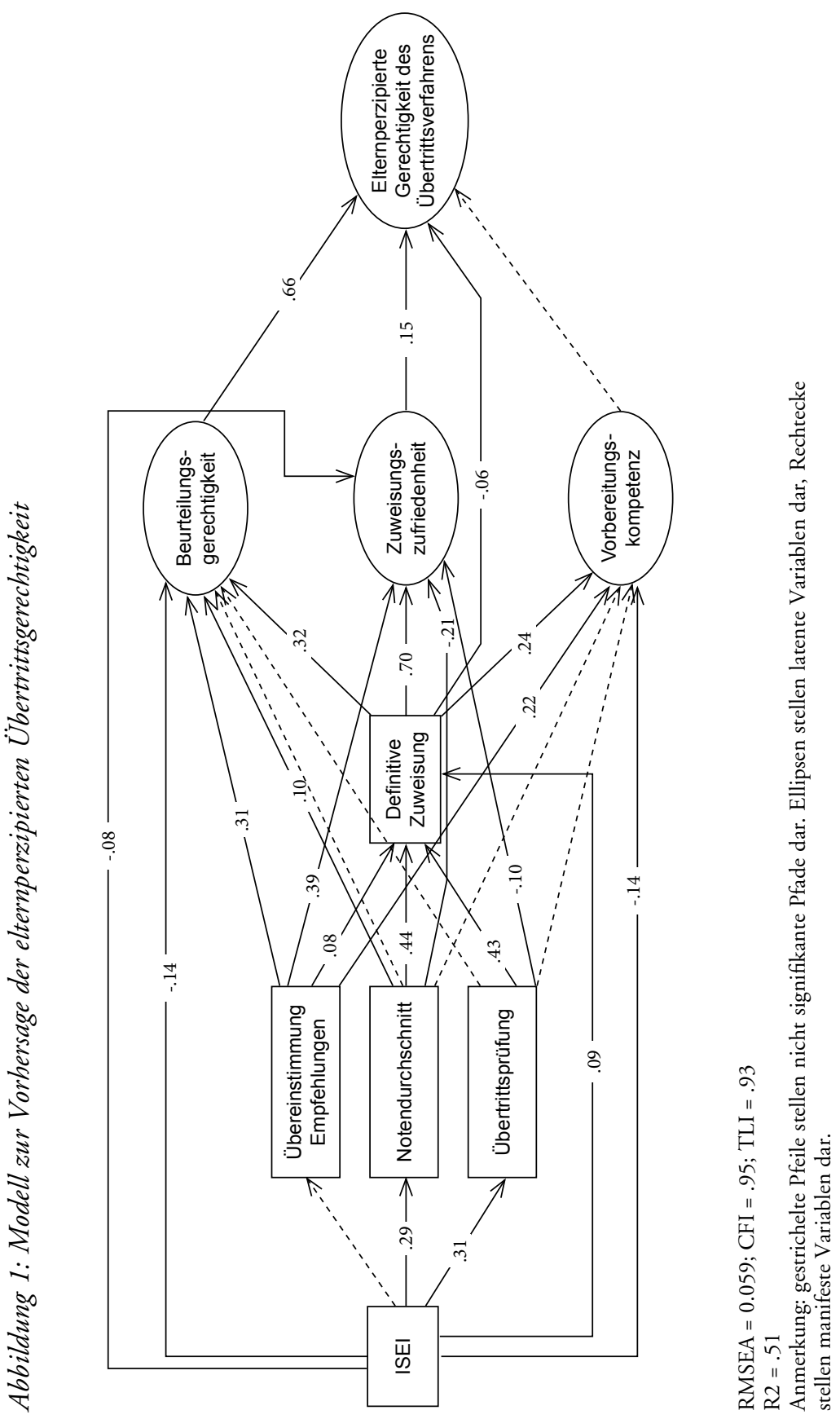


sozioökonomischen Status Mädchen eher in eine höhere Abteilung zugeteilt werden als Jungen. Dieser Effekt verschwand, als zusätzlich die Lehrpersonenempfehlung in die Berechnungen aufgenommen wurde, und ist demnach in der Zuweisungsempfehlung der Lehrpersonen enthalten.

Dass die Elternempfehlung am Ende keinen signifikanten Einfluss mehr auf die definitive Zuweisung hatte, lässt sich auf zwei Fakten zurückführen. Erstens resultierte aus der Übereinstimmung der Lehrpersonempfehlung und der Übertrittsprüfung eine direkte entsprechende Zuweisung. Zweitens gab es eine hohe Übereinstimmung zwischen den Eltern- und den Lehrpersonempfehlungen. Bereits 2007 konnten Trautwein und Baeriswyl zeigen, dass die Lehrpersonempfehlung und der tatsächliche Übergang in die Sekundarstufe I stark zusammenhängen, was die vorliegende Untersuchung bestätigt.

Obwohl die Pseudo- $\mathrm{R}^{2}$ nicht in der derselben Weise interpretiert werden können wie bei linearen Regressionsmodellen, verweisen die Werte der vorliegenden Schätzungen auf eine sehr hohe Modellpassung und einen hohen Erklärungsanteil des Zuweisungsentscheids durch diese reglementarisch vorgesehenen übertrittsrelevanten Prädiktoren. Das kann als ein Hinweis gedeutet werden, dass das Bildungsreglement auch tatsächlich wie vorgesehen in der Praxis angewendet wird.

Zur Vorhersage der elternperzipierten Gerechtigkeit des Übertrittsverfahrens wurde ein Strukturgleichungsmodell analysiert, das neben den zuweisungsrelevanten Variablen elternperzipierte Urteile, nämlich das (un)gerechte Beurteilungsverhalten der Lehrperson, die Zuweisungszufriedenheit der Eltern und die Vorbereitungskompetenz der Lehrperson enthält. Angenommen wurde, dass die zuweisungsrelevanten Variablen Übertrittsprüfung, Notenschnitt und Übereinstimmung der Empfehlungen die elternperzipierten Variablen beeinflussen. Hier war das Differenzmass zwischen der Elternempfehlung und der Lehrpersonempfehlung der wichtigste Einflussfaktor. Unter Kontrolle der Noten und der Übertrittsprüfung zeigt sie starke Zusammenhänge mit Beurteilungsgerechtigkeit, der Zuweisungszufriedenheit und der Vorbereitungskompetenz. Dies bedeutet, wenn die Lehrperson die gleiche oder eine höhere Zuweisung vergibt, als dies die Eltern tun, beurteilen die Eltern das Beurteilungsverhalten der Lehrpersonen als gerechter. Die Eltern schreiben dann der Lehrperson bessere Vorbereitungskompetenzen zu, und die Eltern sind zufriedener mit der Zuweisung. Letzteres wird auch dadurch unterstützt, dass die definitive Zuweisung höher ausfällt, wenn die Lehrperson eine höhere Empfehlung ausspricht. Alle bis auf eine Variable werden in diesem Modell vom ISEI beeinflusst. Je höher der ISEI, desto höher fiel der Notenschnitt aus, umso besser schnitten Kinder in der Übertrittsprüfung ab (vor allem primäre Herkunftseffekte) und desto höher war die definitive Zuweisung (vor allem sekundäre Herkunftseffekte). Weiter bewerteten Eltern mit höherem ISEI das Beurteilungsverhalten der Lehrperson als ungerechter. Sie waren unzufriedener mit der Zuweisung, sprachen der Lehrperson eine geringere Vorbereitungskompetenz zu und schätzten das Übertrittsverfahren insgesamt als 
weniger gerecht ein. Hier zeigt sich, dass Eltern der bildungsnäheren Schichten kritischer gegenüber dem Übertrittssystem und den Lehrpersonen sind.

Den grössten Einfluss auf die von den Eltern wahrgenommene Gerechtigkeit des Übertrittsverfahrens hatte das erlebte Beurteilungsverhalten der Lehrperson. Dies ergänzt vorliegende Befunde zu den positiven Auswirkungen des erlebten gerechten Lehrerhandelns bei Schülerinnen und Schülern (Dalbert, 2011, 2013). Auch bei den Eltern scheint gerechtes Lehrerhandeln Auswirkungen auf globale Urteile zu haben. Als sehr interessant stellt sich die Wirkungsweise der eingeschätzten Vorbereitungskompetenz und der Zufriedenheit mit der Zuweisung dar. Diese beiden Faktoren scheinen nahezu gänzlich in der Beurteilungsgerechtigkeit enthalten zu sein. Diese Resultate erweitern die Arbeit von Baeriswyl et al. (2013) dahin gehend, dass es eben nicht die elternperzipierte Vorbereitungsgüte ist, die den höchsten Einfluss auf die Gerechtigkeitseinschätzung des Übertrittsverfahrens hat, sondern die wahrgenommene Beurteilungsgerechtigkeit. Für eine weitere Aufklärung dieser Befunde sind zusätzliche Analysen notwendig.

Der negative Zusammenhang der definitiven Zuweisung mit der von den Eltern erlebten Gerechtigkeit des Übertrittsverfahrens scheint auf den ersten Blick irritierend. Je höher die definitive Zuweisung des Kindes ist, desto niedriger fällt die Gerechtigkeitseinschätzung des Übertrittsverfahrens aus. Zum Beispiel schätzen Eltern von einem Kind mit einer Zuweisung in die Progymnasialabteilung das Übertrittsverfahren als weniger gerecht ein. Berücksichtigt man die positive Korrelation zwischen Zuweisung und sozioökonomischem Status (siehe Tab. 2, $r=0,35)$, kann man schliessen, dass Eltern aus höheren Schichten kritischer gegenüber Schulsystemen sind. Da höhere Schichten auch bildungsnähere Schichten sind, ist denkbar, dass die Zuweisung aufgrund der Konfundierung mit Bildungsnähe einen Indikator für erhöhte kritische Reflexion darstellt. Auch zu diesem Befund sind weitere Forschungsbemühungen besonders wünschenswert. Denn es finden sich keine Hinweise, dass gerade die Schichten, welche durch sekundäre Herkunftseffekte benachteiligt werden, dies auch so erleben.

\section{Anmerkungen}

1 Universität Freiburg/Schweiz

2 California State University, Fresno

\section{Bibliographie}

Baeriswyl, F. (2013). Verminderung sozialer Ungerechtigkeit bei Schulübergängen. Schaffts ein mehrkriteriales Übertrittsverfahren mit standardisiertem Leistungstest? In D. Deissner (Hrsg.), Chancen bilden. Wege zu einer gerechteren Bildung - ein internationaler Erfahrungsaustausch (S. 153-168). Wiesbaden: Springer VS.

Baeriswyl, F., Wandeler, C., Trautwein, T. \& Oswald, K. (2006). Leistungstest, Offenheit von Bildungsgängen und obligatorische Beratung der Eltern. Reduziert das Deutschfreiburger Übergangsmodell die Effekte des sozialen Hintergrunds bei Übergangsentscheidungen? Zeitschrift für Erziehungswissenschaft, 9, 373-392. 
Baeriswyl, F., Wandeler, C. \& Trautwein, U. (2011). «Auf einer anderen Schule oder bei einer anderen Lehrkraft hätte es fürs Gymnasium gereicht»: Eine Untersuchung zur Bedeutung von Schulen und Lehrkräften für die Übertrittsempfehlung. Zeitschrift für pädagogische Psychologie, 25, 39-47.

Baeriswyl, F., Wandeler, C. \& Biewer, C. (2013). Wie gerecht wirkt das Übertrittsverfahren von der Primarschule in die Sekundarstufe I auf die Eltern? In E. Wannack, S. Bosshart, A. Eichenberger, M. Fuchs, E. Hardegger \& S. Marti (Hrsg.), 4- bis 12-Jährige - Ihre schulischen und ausserschulischen Lern- und Lebenswelten (S. 87-97). Münster: Waxmann.

Baumert, J. \& Schümer, G. (2001). Familiäre Lebensverhältnisse, Bildungsbeteiligung und Kompetenzerwerb. In J. Baumert, E. Klieme, M. Neubrand, M. Prenzel, U. Schiefele, W. Schneider, P. Stanat, K.-J. Tillmann \& M. Weiss (Hrsg.), PISA 2000: Basiskompetenzen von Schülerinnen und Schülern im internationalen Vergleich (S. 323-407). Opladen: Leske + Budrich.

Baumert, J., Artelt, C., Klieme, E., Neubrand, M., Prenzel, M., Schiefele, U., Schneider, W., Tillmann, K. J. \& Weiss, M. (Hrsg.) (2003). PISA 2000. Ein differenzierter Blick auf die Länder der Bundesrepublik Deutschland. Opladen: Leske + Budrich.

Baumert, J., Maaz, K., Gresch, C., McElvany, N., Anders, Y., Jonkmann, K., Neumann, M. \& Watermann, R. (2010). Der Übergang von der Grundschule in die weiterführende Schule - Leistungsgerechtigkeit und regionale, soziale und ethnisch-kulturelle Disparitäten: Zusammenfassung der zentralen Befunde. In K. Maaz, J. Baumert, C. Gresch \& N. McElvany (Hrsg.), Der Übergang von der Grundschule in die weiterführende Schule Leistungsgerechtigkeit und regionale, soziale und ethnisch-kulturelle Disparitäten (S. 5-22). Bonn, Berlin: BMBF.

Becker, R. (2000). Klassenlage und Bildungsentscheidungen. Eine empirische Anwendung der Wert-Erwartungstheorie. Kölner Zeitschrift für Soziologie und Sozialpsychologie, 52, 450-474.

Becker, R. (2003). Educational expansion and persistent inequalities of education. Utilizing subjective expected utility theory to explain increasing participation rates in upper secondary school in the Federal Republic of Germany. European Sociological Review, 19, 1-24.

Becker, R. (2004). Soziale Ungleichheit von Bildungschancen und Chancengerechtigkeit. In R. Becker \& W. Lauterbach (Hrsg.), Bildung als Privileg? Erklärungen und Befunde zu den Ursachen der Bildungsungleichheit (S. 161-193). Wiesbaden: VS Verlag für Sozialwissenschaften.

Becker, R. (2011). Entstehung und Reproduktion von Bildungsungleichheiten. In R. Becker (Hrsg.), Lehrbuch der Bildungssoziologie (S. 87-138). Wiesbaden: VS Verlag für Sozialwissenschaften.

Becker, R. \& Schubert, F. (2011). Die Rolle von primären und sekundären Herkunftseffekten für Bildungschancen von Migranten im deutschen Schulsystem. In R. Becker (Hrsg.), Integration durch Bildung (S. 161-194). Wiesbaden: VS Verlag für Sozialwissenschaften.

Boudon, R. (1974). Education, Opportunity, and Social Inequality: Changing Prospects in Western Society. New York: Wiley.

Bos, W., Lankes, E.-M., Prenzel, M., Schwuppert, K., Walther, G. \& Valtin, R. (Hrsg.). (2003). Erste Ergebnisse aus IGLU. Schülerleistungen am Ende der vierten Jahrgangsstufe im internationalen Vergleich. Münster: Waxmann.

Dalbert, C. (2011). Warum die durch SchülerInnen individuell und subjektiv erlebte Gerechtigkeit des Lehrerhandelns wichtig ist. Zeitschrift für Pädagogische Psychologie, 25 (1), 5-18.

Dalbert, C. (Hrsg.). (2013). Gerechtigkeit in der Schule. Wiesbaden: Springer VS.

Dalbert, C. \& Stoeber, J. (2006). The personal belief in a just world and domain-specific beliefs about justice at school and in the family: A longitudinal study with adolescents. International Journal of Behavioral Development, 30, 200-207.

Ditton, H. (2007). Kompetenzaufbau und Laufbahnen im Schulsystem. Ergebnisse einer Längsschnittuntersuchung an Grundschulen. Münster: Waxmann. 
Ditton, H. (2008): Durchlässigkeit an den Übergängen. In: Robert Bosch Stiftung (Hrsg.), Zukunftsvermögen Bildung (S. 53-56). Stuttgart.

Ditton, H. \& Krüsken, J. (2006). Der Übergang von der Grundschule in die Sekundarstufe I. Zeitschrift für Erziehungswissenschaft, 9, 348-372.

Ditton, H., Krüsken, J. \& Schauenberg, M. (2005). Bildungsungleichheit - der Beitrag von Familie und Schule. Zeitschrift für Erziehungswissenschaft, 8, 285-303.

Enders, C. K. \& Bandalos, D. L. (2001). The relative performance of full information maximum likelihood estimation for missing data in structural equation models. Structural Equation Modeling: A Multidisciplinary Journal, 8(3), 430-457.

Fend, H. (1974). Gesellschaftliche Beziehungen schulischer Sozialisation. Weinheim, Basel: Beltz.

Fischer, D. (2007). Einleitung: Gerechtigkeit im Bildungssystem. In D. Fischer \& V. Elsenbast (Hrsg.), Zur Gerechtigkeit im Bildungssystem (S. 7-14). Münster, New York, München, Berlin: Waxmann.

Ganzeboom, H. B. G., Treiman, D. J. (1996). Internationally comparable measures of occupational status for the 1988 international standard classification of occupations. Social Science Research, 25, 201-239.

Jonkmann, K., Maaz, K., Neumann, M. \& Gresch, C. (2010). Übergangsquoten und Zusammenhänge zu familiärem Hintergrund und schulischen Leistungen: Deskriptive Befunde. In K. Maaz, J. Baumert, C. Gresch \& N. McElvany (Hrsg.), Der Übergang von der Grundschule in die weiterführende Schule - Leistungsgerechtigkeit und regionale, soziale und ethnisch-kulturelle Disparitäten (S. 125-152). Bonn, Berlin: BMBF.

Kanders, M. (2000). Das Bild der Schule aus Sicht der Schüler und Lehrer II. Dortmund: IFS-Verlag.

Maaz, K., Hausen, C., McElvany, N. \& Baumert, J. (2006). Stichwort: Übergänge im Bildungssystem Theoretische Konzepte und ihre Anwendung in der empirischen Forschung beim Übergang in die Sekundarstufe. Zeitschrift für Erziehungswissenschaft, 9 (3), 299-327.

Maaz, K. \& Nagy, G. (2009). Der Übergang von der Grundschule in die weiterführenden Schulen des Sekundarschulsystems: Definition, Spezifikation und Quantifizierung primärer und sekundärer Herkunftseffekte. Zeitschrift für Erziehungswissenschaft, Sonderheft 12, 153-182.

Maaz, K., Baumert, J. \& Trautwein, U. (2009). Genese sozialer Ungleichheit im institutionellen Kontext der Schule: Wo entsteht und vergrössert sich soziale Ungleichheit? In K. Maaz, J. Baumert, C. Gresch \& N. McElvany (Hrsg.), Der Übergang von der Grundschule in die weiterführende Schule - Leistungsgerechtigkeit und regionale, soziale und ethnisch-kulturelle Disparitäten (S. 27-64). Bonn, Berlin: BMBF.

Maaz, K., Baeriswyl, F. \& Trautwein, U. (2011). Herkunft zensiert. Leistungsdiagnostik und soziale Ungleichheiten in der Schule. Vodafone Stiftung Deutschland.

Maier, G., Streicher, B., Jonas, E. \& Woschée, R. (2007). Gerechtigkeitseinschätzungen in Organisationen: Die Validität einer deutschsprachigen Fassung des Fragebogens von Colquitt (2001). Diagnostica, 53, 97-108.

McKelvey, R. D. \& Zavoina, W. (1975). A statistical model for the analysis of ordinal level dependent variables. Journal of Mathematical Sociology, 4, 103-20.

Mikula, G. (1980). Einleitung: Thematische Schwerpunkte der psychologischen Gerechtigkeitsforschung. In G. Mikula (Hrsg.), Gerechtigkeit und soziale Interaktion. Experimentelle und theoretische Beiträge aus der psychologischen Forschung (S. 13-24). Bern, Stuttgart, Wien: Hans Huber.

Muthén, L. K., \& Muthén, B. O. (1998-2011). Mplus User's Guide. Sixth Edition. Los Angeles, CA: Muthén \& Muthén.

Neuenschwander, M. P. \& Grunder, H.-U. (2010). Schulübergang und Selektion. Chur: Rüegger. 
Peter, F., Donat, M., Umlauft, S. \& Dalbert, C. (2013). Einführung in die Gerechtigkeitspsychologie. In C. Dalbert (Hrsg.), Gerechtigkeit in der Schule (S.11-32). Wiesbaden: Springer VS.

Raykov, T. (2005). Analysis of longitudinal studies with missing data using covariance structure modeling with full-information maximum likelihood. Structural Equation Modeling, 12, 493-505.

Schmitt, M. (1993). Abriss der Gerechtigkeitspsychologie (Berichte der Arbeitsgruppe "Verantwortung, Gerechtigkeit, Moral», Nr. 70). Trier. Universität Trier, Fachbereich I - Psychologie.

Snijders, T. A. B. \& Bosker, R. J. (2012). Multilevel analysis. An introduction to basic and advanced multilevel modeling. London: Sage Publications.

Trautwein, U. \& Baeriswyl, F. (2007). Wenn leistungsstarke Klassenkameraden im Nachteil sind. Referenzgruppeneffekte bei Übertrittsentscheidungen. Zeitschrift für Pädagogische Psychologie, 21 (2), 119-133.

Trautwein, U., Baeriswyl, F., Lüdtke, O. \& Wandeler, C. (2008). Die Öffnung des Schulsystems: Fakt oder Fiktion? Empirische Befunde zum Zusammenhang von Grundschulübertritt und Übergang in die gymnasiale Oberstufe. Zeitschrift für Erziehungswissenschaft, 11, 648-665.

Tyler, P. (1962). Children's evaluations of the characteristics of a good teacher. British Journal of Educational Psychology, 32, 258-266.

UCLA: Statistical Consulting Group. (2013). Zugriff am 27.05.2013 unter http://statistics. ats.ucla.edu/stat/mult_pkg/faq/general/Psuedo_RSquareds.htm

Schlagworte: Soziale Disparitäten, Gerechtigkeitserleben, Übertritt, Primärer und Sekundärer Herkunftseffekt, Zuweisungsempfehlung

\section{Les effets de l'origine sociale et les perceptions de justice pendant la transition de l'école primaire à l'école secondaire}

\section{Ré sumé}

La partie germanophone du canton de Fribourg utilise pour le passage de l'école primaire à l'école secondaire un système composé des notes des élèves, des évaluations de leurs habilités cognitives, des recommandations des professeurs et des parents et d'un examen en allemand et en mathématique. Les cohortes 2009 et 2010 ont été évaluées. Les données ont permis une séparation des effets de l'origine sociale et ont montré l'influence de l'origine des élèves sur la décision de passage. Même lorsqu'on contrôle les effets des recommandations des parents et des professeurs, l'effet d'origine reste. Les perceptions de justice des parents concernant le système de transition sont aussi influencées par leur origine sociale. Elles s'expliquent toutefois avant tout par la perception de la justice de l'enseignant-e.

Mots-clés: Disparité sociale, perception de justice, effet d'origine sociale, transition 


\section{Effetti d'origine e percezione della giustizia nel passaggio dalla scuola primaria a quella di grado secondario I}

\section{Rissasunto}

Nella parte germanofona del Cantone di Friburgo/CH viene utilizzata una procedura multicriterio per il passaggio dalla scuola primaria a quella di grado secondario I. Oltre alle note scolastiche, alla valutazione delle capacità cognitive da parte di docenti, nonché alle loro raccomandazioni e a quelle dei genitori, viene svolta un' ulteriore prova di confronto in tedesco e matematica. In due ampi rilevamenti, sono stati intervistati dettagliatamente allieve e allievi delle coorti 2009 e 2010, i loro genitori, nonché i e le loro docenti. Separando empiricamente gli effetti d'origine primari e secondari i dati evidenziano l'influenza dell'origine sociale su questo passaggio. Anche controllando la prestazione, le note scolastiche e le raccomandazioni dei genitori e dei e delle docenti, l'influenza dell'origine sociale rimane significativa. Gli effetti d'origine secondari non si riflettono nella percezione di giustizia dei genitori appartenenti a un ceto socioeconomico basso, mentre sono tendenzialmente i genitori appartenenti a ceti sociali alti a percepire ingiustizie in tale passaggio. In generale, la giustizia percepita dai genitori della parte germanofona di Friburgo in merito alla transizione dei propri figli, viene spiegata soprattutto dalla loro percezione del grado di giustizia degli insegnanti.

Parole chiave: Disparità sociali, percezione della giustizia, passaggio, effetti d'origine primari e secondari, raccomandazione

\section{Effects of social origin and the justice perception during the transition from primary school to secondary school}

\section{Abstract}

In the German-speaking part of the canton Freiburg/CH 6th grade students transition into a tracked middle school. Students are allocated to ability tracks by a process that takes into account school marks, the appraisals of cognitive abilities, the allocation recommendation of the teacher and the parents, and a standardized test in German and Math. The data from 1685 students of the cohorts of 2009 and 2010 allowed the empirical separation of primary and secondary social origin effects in the context of the transition decisions. Even if the allocation recommendations of the parents and the teachers were used as control variables the effects of social origin persisted. The secondary social origin effects were not reflected in the perceived educational justice of the affected parents with lower socio-economic background. However, parents who perceived educational injustice tended to come from a higher socio-economic background. The parental perceived justice of the transition system is mainly influenced by 
perceived teacher justice, however to a smaller extent origin dependent criteria were also relevant.

Keywords: Social inequality, justice perception, effects of social origin, transition, transition recommendation, educational justice 